\title{
Quantifying Canadians' use of the Internet as a source of information on behavioural risk factor modifications related to cancer prevention
}

\author{
C. G. Richardson, PhD; L. G. Hamadani, MPH; C. Gotay, PhD
}

This article has been peer reviewed.

\begin{abstract}
Introduction: The purpose of this study was to quantify the frequency and timing of Canadians' Internet searches for information on modifying cancer prevention-related behavioural risk factors.
\end{abstract}

Methods: We used the Google AdWords Keyword tool to estimate the number of Internet searches in Canada from July 2010 to May 2011 for content associated with the keywords "physical activity / exercise," "healthy eating / weight loss" and "quit smoking."

Results: For “physical activity / exercise,” 663 related keywords resulted in 117951699 searches. For "healthy eating / weight loss,” 687 related search terms yielded 98277954 searches. "Quit smoking” was associated with 759 related keywords with 31688973 searches. All search patterns noticeably peaked in January 2011.

Conclusion: Many Canadians are actively searching for information on the Internet to support health behaviour change associated with cancer prevention, especially during the month of January. To take advantage of this opportunity, key stakeholders in cancer prevention need to identify knowledge translation priorities and work with health agencies to develop evidence-based strategies to support Internet-facilitated behaviour change.

Keywords: cancer, prevention, internet, weight loss, physical activity, smoking, healthy eating

\section{Introduction}

Tobacco use, alcohol use, overweight, an unhealthy diet and a sedentary lifestyle have been identified as the primary modifiable risk factors for cancer, ${ }^{1-3}$ and more than $30 \%$ of cancers could be avoided by making relevant lifestyle changes to avoid these risk factors. ${ }^{4}$ This potential for cancer prevention has led many health agencies to prioritize activities that contribute to starting and maintaining behaviour changes related to reducing tobacco use and exposure, reducweight and obesity, improving diets and increasing physical activity. ${ }^{4-6}$

One of the most common methods that members of the public use to look for current health-related information is through Internet searches. ${ }^{7}$ According to a Statistics Canada report, $80 \%$ of Canadians aged 16 years and older (21.7 million people) used the Internet for personal reasons in 2009, an $8 \%$ increase from 2007 and $12 \%$ increase from $2005 .{ }^{8,9}$ In addition, searches for information on specific diseases ing alcohol consumption, reducing over- or lifestyle factors increased by $11 \%$, with $70 \%$ of Canadian home Internet users reporting that they use the Internet to search for health information, compared with 59\% in 2007., Although this increase may be part of a general trend towards seeking information on the Internet, the widely publicized emergence of the H1N1 influenza strain as a global pandemic in June $2009^{10}$ may have contributed to this. The widespread use of the Internet by members of the public has led some health researchers to monitor changes in Internet-based information-seeking activity as a means of tracking changes in health behaviours, health status and public attitudes towards health promotional activities. ${ }^{11}$

One of the first steps towards taking advantage of the opportunity to disseminate information to the public efficiently and effectively is to investigate the search patterns used by people seeking health information (i.e. timing of the searches and popular search terms used). Although there are many different search engines, the one developed and maintained by Google has come to dominate Internet searching for more than five years. Of the 137 billion estimated total searches performed in the United States in 2008, 85 billion used Google; similar numbers apply to searches conducted worldwide. ${ }^{12}$ Google domains (i.e. google.ca and google.com) rank as the primary search engine in Canada, capturing $81 \%$ of the total volume of Canadian searches for the 12-week period ending July 2, 2011. ${ }^{13}$

The purpose of this study was to determine the frequency and timing of Internet

\section{Author references:}

School of Population and Public Health, University of British Columbia, British Columbia, Canada

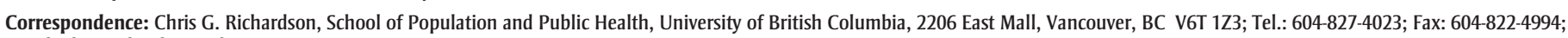
Email: chris.richardson@ubc.ca 
searches for information on the following modifiable risk factors associated with cancer prevention: sedentary behaviour, being overweight and smoking.

\section{Methods}

We used the Google AdWords Keyword tool $^{14}$ and Google Insight ${ }^{15}$ to get data on the frequency and timing of searches for three separate sets of search terms. The Keyword tool is a free online tool maintained by Google. After entering one or more search terms, the tool generates a list of all the Google-identified relevant keywords used to retrieve related information. The Keyword tool also provides average global and local search volumes associated with each term for the previous 12 months as well as the number of searches conducted each month for the past 11 months. The tool also allows users to narrow down the search for relevant keywords by country, language and category (e.g. health, business, etc.)
We conducted separate searches on July 14, 2011, for the period July 2010 to May 2011 for each of the following three English terms: "physical activity / exercise” (for sedentary behaviour), "healthy eating / weight loss"' (for overweight and obesity) and "quit smoking” together with "Canada," “all languages” and "all categories." Three independent reviewers (C.R., L.H., C.G.) with expertise in public health and cancer prevention evaluated the keywords Google associated with each term and reached consensus on the terms to include in the frequency calculations. They deleted those terms that did not appear to be directly related to health behaviours associated with cancer prevention from the results. When there was doubt about a specific keyword, the term was entered directly into the Google search engine and the search results investigated. Because $90 \%$ of users only look for information in the first three pages of their Internet search results, ${ }^{16}$ we removed any search terms that did not generate information about behaviours related to cancer prevention on the first three pages of the search results.

Google Insight also provided search trends from 2004 to 2011 for our three search terms. Google Insight characterizes the search volume patterns across regions, categories and time frames. Although the trends include searches for the main keywords, Google Insight does not provide absolute frequencies. Rather, the total number of monthly searches are standardized using the month with the highest number of searches as a reference (i.e. the frequency of searches for individual months are presented as a percentage of the month with the highest number of searches)..$^{17}$

\section{Results}

The Google AdWords Keywords search using the terms "physical activity / exercise” resulted in 799 unique terms.

FIGURE 1

Estimated number of monthly searches from July 2010 to May 2011 (top row) and search trends from January 2004 to January 2011 (bottom row) for the search term "physical activity / exercise"

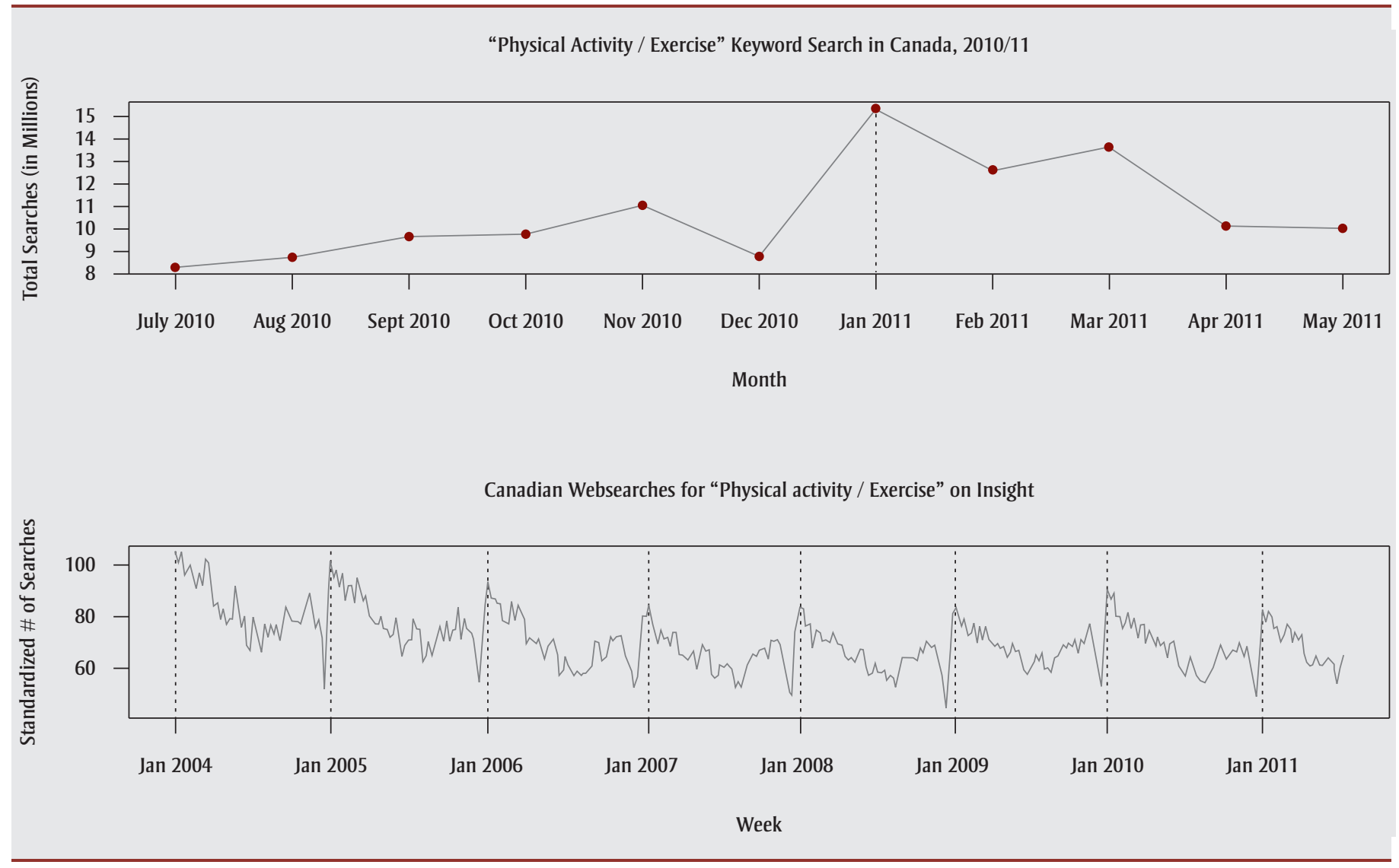


After removing those not related to cancer prevention (e.g. "kegel exercises"), there remained 663 terms with a total of 117951699 searches over the 11month period. The three most popular phrases were "LA Fitness" (a Californiabased fitness program with gyms in Canada and the United States; $17 \quad 620 \quad 000$ searches), "how to exercise" (11909000 searches) and "why exercise”' (11909000 searches). Monthly searches peaked in January 2011 (15 $333 \quad 720$ searches), accounting for $13 \%$ of all the searches in the study period, while the lowest number of searches took place in July 2010 (see Figure 1, top row). The results from the Google Insight search showed similar peaks in January every year between 2004 and 2011 (see Figure 1, bottom row).

The Google AdWords Keywords search using the terms "healthy eating / weight loss" resulted in 803 unique terms. After these were reviewed and unrelated terms eliminated (e.g. "dinner recipes" and “diabetic diet”), there remained 687 search terms related to health behaviours associated with cancer prevention with 98277954 search queries for the 11month period, July 2010 to May 2011. The three most popular keywords were "LA Weight Loss" (a California-based weight loss program; 15868000 searches), "weight loss" (7 934000 searches) and "how to lose weight" (7 415000 searches). The peak for all searches was $13.8 \%$, in January $2011 \quad\left(\begin{array}{lll}13 & 529 & 551\end{array}\right.$ searches), while July 2010 had the fewest, at $6.8 \%$ (see Figure 2, top row). Google Insight showed a similar peak every January from 2004 to 2011. We also observed another distinctive peak during the week of May 23 to 29, 2010 (see Figure 2, bottom row).

The Google AdWords Keywords search using the keywords "quit smoking" yielded 793 unique search terms. Of these, we deleted 34 unrelated terms (e.g. "smoking weed"). The remaining 759 search terms yielded 31688973 search queries for the 11-month period. The three most popular search terms were "how to quit smoking”' (842 500 searches), "why quit smoking” (842 500 searches) and "I quit smoking”' (842 500 searches). The highest proportion of search traffic $(13.5 \%$ of all the searches) was in January 2011 (see Figure 3, top row). Google Insight showed a similar peak in January of each year from 2004 to 2011 (see Figure 3, bottom row).

\section{Discussion}

The results of this study show that many Canadians search the Internet for information on modifying lifestyle factors that have been linked to cancer. Of our chosen search terms, "physical activity / exercise" (for sedentary behaviour) had the highest number of searches, followed by "healthy eating / weight loss", (for overweight/obesity) and "quit smoking." All these search terms showed temporal

FIGURE 2

Estimated number of monthly searches from July 2010 to May 2011 (top row) and search trend from January 2004 to January 2011 (bottom row) for the search term "healthy eating / weight loss"

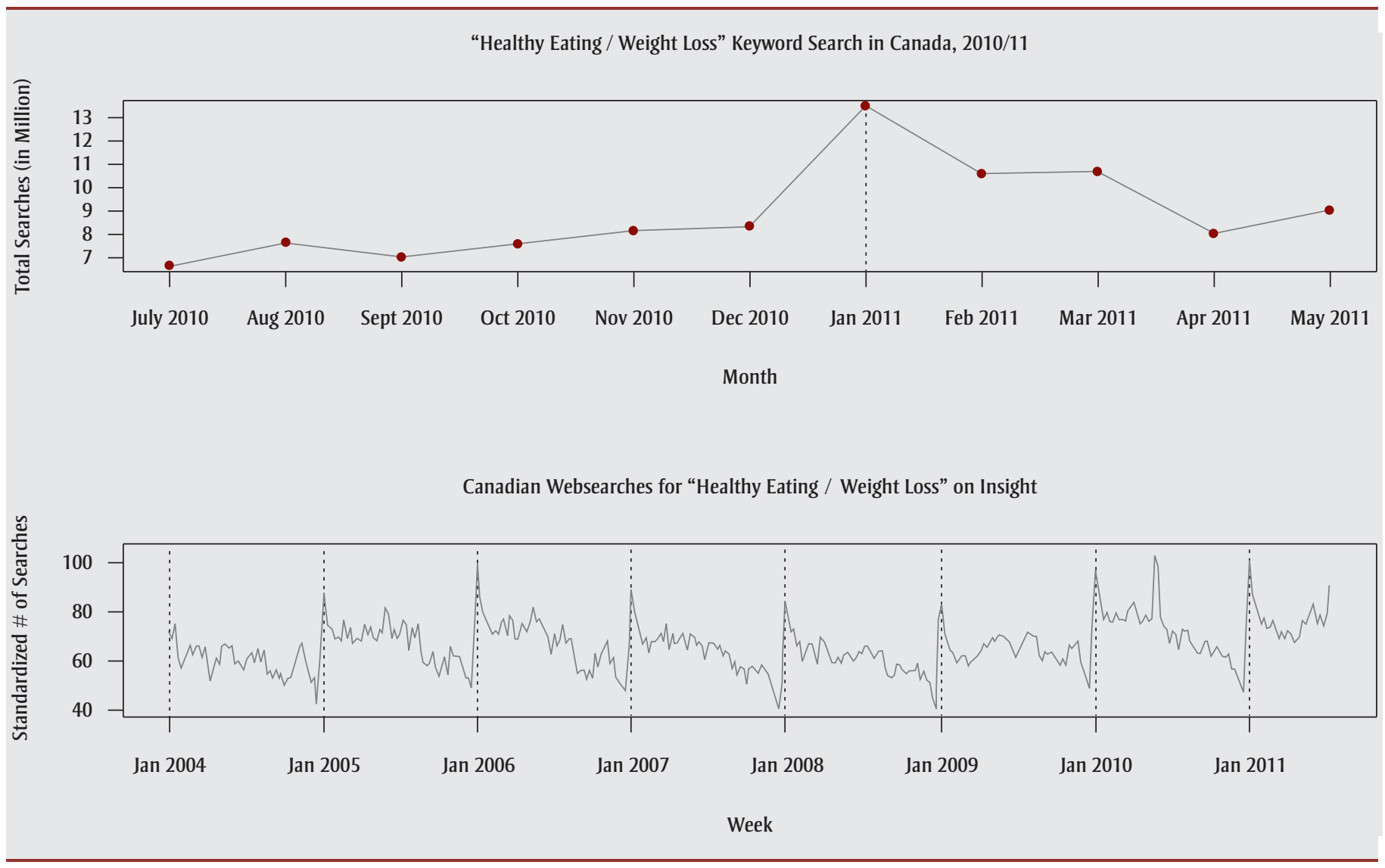


FIGURE 3

Estimated number of monthly searches from July 2010 to May 2011 (top row) and search trend from January 2004 to January 2011 (bottom row) for the search term "quit smoking"

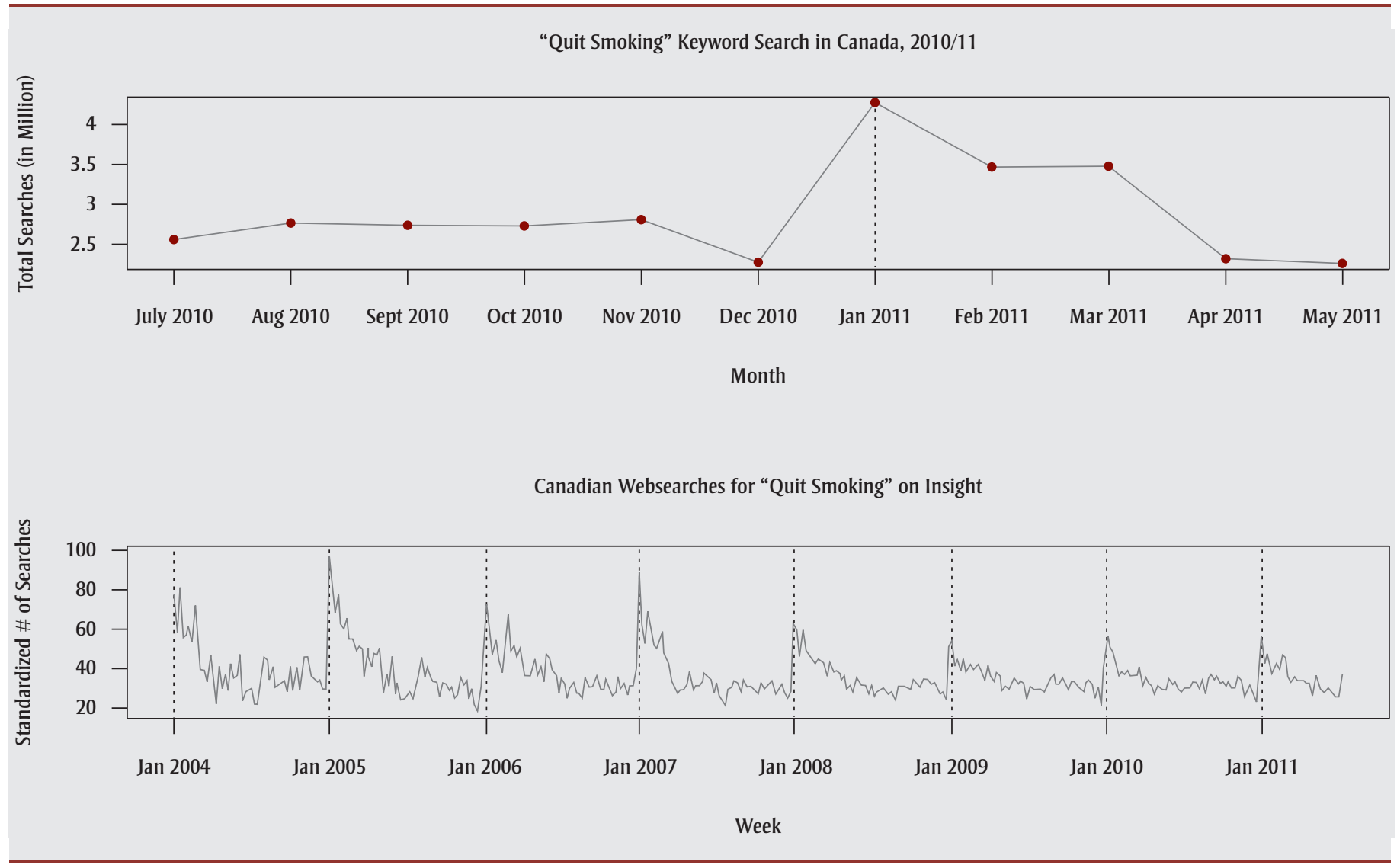

effects: specifically, distinct increases in search traffic during the month of January. We speculate that this pattern is tied to setting New Year's resolutions-previous studies have found that almost $50 \%$ of Americans initiate health-related behaviour changes involving weight loss, smoking cessation and/or exercising at this time. ${ }^{18}$ This phenomenon could represent a promising opportunity to implement seasonally tailored Internet-based health campaigns and interventions.

We suspect that the high volume of searches for "LA Fitness" for "physical activity / exercise” represent searches for the multinational chain of private fitness gyms that was using an Internet-based advertising campaign to promote its business. ${ }^{19}$ We also observed a distinctive peak for "healthy eating / weight loss" in the week of May 23 to 29, 2010 (see Figure 2, bottom row). We could not identify any health promotion events ${ }^{20}$ or news headlines ${ }^{21}$ during that week to explain the spike in the number of searches. However, the finale of the Season 9 of "The Biggest Loser," a television reality show that features obese people competing for cash prizes by losing high percentages of their initial weight, aired on May 25, 2010 to approximately 9.4 million viewers. ${ }^{22}$ While the sharp rise in the searches for "healthy eating / weight loss" may have been related to this broadcast, it is also possible that the increase in search activity may represent a seasonal trend. Further research is needed to confirm this as a regular opportunity to promote cancer prevention-related health behaviours.

The large number of Canadians (more than 15 million in 2009) ${ }^{8}$ actively searching the Internet for health-related information represents a valuable opportunity to support those modifiable risk factors that are relevant to cancer prevention. Online health information has demonstrated the potential to influence behaviour. For example, more than $70 \%$ of Internet users report that the health information they find online influences a treatment decision. ${ }^{7,9}$ Moreover, access to reliable information is linked to "reduced anxiety, increased feelings of self-efficacy, and a decrease in utilization of ambulatory care." ${ }^{23}$ Although a great deal of useful health information is available on the Internet, a substantial portion of the content may not be evidence-based. For example, much of the content on stopping smoking available on the video-sharing website, YouTube.com, is not derived from evidence-based cessation strategies. $^{24}$

\section{Strengths and limitations}

There are several limitations associated with this research. First, the monthly search numbers are estimates provided by Google and do not reflect the exact number of searches for each term. Second, it is not possible to identify how many 
different individuals conducted the searches; some individuals may be responsible for multiple searches, while others may have conducted a single search. In some cases, we were unable to determine if a related search term was used to obtain specific information on the concept under investigation. For example, some people typing in the search term "weight loss" may not necessarily be searching for information on how to lose weight but for explanations for sudden weight loss. However, most terms, especially those with high frequencies, did appear to be directly relevant to this investigation. In addition, the three sets of keywords examined in this study did not include content related to other modifiable risk factors relevant to cancer prevention, for example, reducing alcohol use and exposure to ultraviolet and ionizing radiation, and occupational exposures. Further research is needed to investigate the search activity related to these and other modifiable risks factors.

\section{Conclusion}

The continued growth of the Internet in terms of accessibility and content represents a rapidly expanding opportunity for cancer prevention agencies to disseminate evidence-based information and resources. Online interventions addressing health issues such as smoking, nutrition and physical activity are increasingly popular; ${ }^{25,26}$ the keywords and the related terms examined were associated with more than 240 million searches in 2010 alone. The growing popularity of new Internet interfaces (e.g. smartphone and tablet applications) represents additional opportunities to reach more people in interactive ways. The identified trends suggest that it is worthwhile exploring ways of tailoring online content about physical activity, smoking cessation and healthy eating to specific times of the year, for example, via the Live Well program run by the Canadian Cancer Society, Key stakeholders in cancer prevention, for example, the Canadian Cancer Society, Canadian Partnership Against Cancer and British Columbia Cancer Agency, need to identify Internet knowledge translation priorities and work with community health agencies and provincial health authorities to develop evidence-based strategies to support behaviour change linked to modifiable risk factors for cancer.

\section{References}

1. Colditz GA, Wolin KY, Gehlert S. Applying what we know to accelerate cancer prevention. Sci Transl Med. 2012 Mar 28;4(127):127rv4.

2. Khan N, Afaq F, Mukhtar H. Lifestyle as risk factor for cancer: evidence from human studies. Cancer Lett. 2010 Jul 28;293(2):133-43.

3. Stein CJ, Colditz GA. Modifiable risk factors for cancer. Br J Cancer. 2004 Jan 26;90(2):299-303

4. World Health Organization. Cancer: Fact sheet no. 297 [Internet]. Geneva $(\mathrm{CH})$ : WHO; 2012 Feb [cited 2012 Aug 3]. Available from: http://www.who.int /mediacentre/factsheets/fs297/en/

5. World Health Organization. Cancer control: knowledge into action. WHO guide for effective programmes. Prevention [Internet]. Geneva (CH): WHO; 2007 [cited 2012 Aug 3]. Available from: http://www.who.int/cancer /publications/cancer_control_prevention/en /index.html

6. What is a risk factor [Internet]. Toronto $(\mathrm{ON})$ : Canadian Cancer Society; [modified 2009 Dec 13; cited 2012 Aug 3]. Available from: http: //www.cancer.ca/Canada-wide/Prevention /What \%20is \% 20a \%20risk \%20factor.aspx ?sc_lang $=$ en

7. Fox S, Jones S. The social life of health information [Internet]. Washington (DC): Pew Research Center; 2009 Jun [cited 2012 Aug 3]. Available from: http://www .pewinternet.org/Reports/2009/8-The-Social -Life-of-Health-Information.aspx

8. Statistics Canada. Canadian Internet use survey - 2009 [Internet]. Ottawa (ON): Statistics Canada; [modified 2011 Jul 5; cited 2011 Jul 20]. Available from: http: //www.statcan.gc.ca/daily-quotidien/100510 /dq100510a-eng.htm

9. Underhill C, Mckeown L. Getting a second opinion: health information and the Internet. Health Rep. 2008 Mar;19(1):65-9.
10. Chan M. World now at the start of 2009 influenza pandemic [Internet]. Geneva (CH): WHO; 2009 Jun 11 [cited 2012 Jul 13]. Available from: http://www.who.int /mediacentre/news/statements/2009/h1n1 _pandemic_phase6_20090611/en/index.html

11. Cline RJ, Haynes KM. Consumer health information seeking on the Internet: the state of the art. Health Educ Res. 2001 Dec 1;16(6):671-92.

12. Radwanick $S$. The 2008 digital year in review [Internet]. Reston (VA): comScore; 2009 Jan 30 [cited 2012 Aug 3]. Available from: http://www.comscore.com/Press_Events /Presentations_Whitepapers/2009/2008 _Digital_Year_in_Review

13. Experian Hitwise. Main Data Center: Top 20 sites and engines [Internet]. Costa Mesa (CA): Experian; [cited 2011 Jul 15]. Available from: http://www.hitwise.com /ca/datacenter/main/dashboard-10557.html

14. Google Adwords [Internet]. Mountain View (CA): Google; 2010 [cited 2012 Aug 3]. Available from: https://accounts.google.com /ServiceLogin?service $=$ adwords\&hl $=$ en_CA \&ltmpl $=\mathrm{jfk} \&$ continue $=$ https $: / /$ adwords .google.ca/um/gaiaauth?apt \%3DNone $\% 26 \mathrm{ltmpl} \% 3 \mathrm{Djfk} \& \mathrm{~cd}=$ CA\&passive $=86400$ $\&$ sacu $=1 \&$ sarp $=1$

15. Google Insights for Search [Internet]. Mountain View (CA): Google; 2010 [cited 2012 Aug 3]. Available from: http://support .google.com/trends/bin/answer.py?hl = en \&answer $=96693$

16. IProspect Search Engine User Behavior Study [Internet]. Boston (MA): iProspect; 2006 Apr[cited 2011 Jul 20]. Available from: http://district4.extension.ifas.ufl.edu/Tech /TechPubs/WhitePaper_2006_SearchEngine UserBehavior.pdf

17. Is the data normalized? [Internet]. Mountain View (CA): Google; [cited 2011 Jun 8]. Available from: http://www.google.com /support/insights/bin/bin/answer.py?answer $=87284 \& \& \mathrm{hl}=$ en

18. Norcross JC, Mrykalo MS, Blagys MD. Auld lang Syne: success predictors, change processes, and self-reported outcomes of New Year's resolvers and nonresolvers. J Clin Psychol. 2002;58(4):397-405. 
19. LA Fitness times email marketing campaign to perfection [Internet]. Brighton (UK): Pure360; [cited 2011 Aug 3]. Available from: http://www.pure360.com/customer-success /case-studies

20. Calendar of health promotion days - 2010 [Internet]. Ottawa (ON): Health Canada; [cited 2011 Aug 3]. Available from: http:// www.hc-sc.gc.ca/ahc-asc/calend/index-eng .php\#may

21. May 2010: portal: current events [Internet]. Los Angeles (CA): Wikipedia; [cited 2011 Aug 3]. Available from: http://en.wikipedia .org/wiki/May_2010

22. The Biggest Loser (U.S. TV series) [Internet]. Los Angeles (CA): Wikipedia; [cited 2011 Aug 3]. Available from: http: //en.wikipedia.org/wiki/The_Biggest_Loser _(U.S._TV_series) \#cite_note-0910season-24

23. Ybarra ML, Suman M. Help seeking behavior and the Internet: a national survey. Int J Med Inform. 2006 Jan;75(1):29-41.

24. Richardson CG, Vettese L, Sussman S, Small SP, Selby P. An investigation of smoking cessation video content on YouTube. Subst Use Misuse 2011;46(7): 893-7.

25. Oenema A, Tan F, Brug J. Short-term efficacy of a web-based computer-tailored nutrition intervention: main effects and mediators. Ann Behav Med. 2005 Feb;29(1):54-63.

26. Van den Berg MH, Schoones JW, Vliet Vlieland TP. Internet-based physical activity interventions: a systematic review of the literature. J Med Internet Res. 2007 Sep 30;9(3):e26. 